\title{
Du CELIB à la région Bretagne : réussite et limites d'une affirmation identitaire
}

\author{
Jacqueline Sainclivier
}

\section{(C) OpenEdition}

Édition électronique

URL : http://journals.openedition.org/abpo/1180

DOI : $10.4000 / a b p o .1180$

ISBN : 978-2-7535-1496-6

ISSN : $2108-6443$

Éditeur

Presses universitaires de Rennes

Édition imprimée

Date de publication : 20 décembre 2004

Pagination : 103-116

ISBN : 978-2-7535-0082-2

ISSN : 0399-0826

\section{Référence électronique}

Jacqueline Sainclivier, «Du CELIB à la région Bretagne : réussite et limites d'une affırmation identitaire ", Annales de Bretagne et des Pays de l'Ouest [En ligne], 111-4 | 2004, mis en ligne le 20 décembre 2006, consulté le 02 mai 2019. URL : http://journals.openedition.org/abpo/1180 ; DOI : $10.4000 / a b p o .1180$ 


\title{
Du CELIB à la région Bretagne : réussite et limites d'une affirmation identitaire
}

\author{
Jacqueline SAINCLIVIER \\ Professeur d'histoire contemporaine \\ CRHISCO - Université Rennes 2 Haute-Bretagne
}

La Seconde Guerre mondiale a laissé en Bretagne de profondes traces physiques et psychologiques. Les ports de Brest, Lorient, Saint-Malo et Saint-Nazaire sont totalement détruits, Rennes et Nantes ont été sérieusement endommagées; si la région a été libérée à l'été 1944, les régions de Lorient et de Saint-Nazaire ne l'ont été qu'après la capitulation allemande de mai 1945. À ces traces physiques s'ajoutent les conséquences psychologiques communes à l'ensemble des Français, dues à l'attitude observée pendant l'occupation (résistance, collaboration, " attentisme ", etc.) suivie de l'épuration, mais aussi aux spécificités du mouvement breton ${ }^{1}$. La région est à reconstruire et le matériel pour l'industrie comme pour l'agriculture est usé et à renouveler totalement; le rationnement accentue le sentiment de dépendance des citadins vis-à-vis des paysans, du moins jusqu'en 1949, date de la fin du rationnement. Toute l'économie est à repenser.

La guerre a contraint à un brassage de population, nombre de Bretons ont découvert d'autres conditions de vie et de travail par le contact avec les réfugiés du nord de la France, par leur séjour en Allemagne ${ }^{2}$. Au lendemain de la Seconde Guerre mondiale, la Bretagne ou plutôt ses élites et une partie de la population prend conscience alors de son retard par rapport au reste de la France. La parution de l'ouvrage de Jean-François Gravier au titre provocateur Paris et le désert français ${ }^{3}$ secoue d'autant plus la Bretagne

1. À l'intérieur du mouvement breton, une fraction des autonomistes a été non seulement vichyssoise mais a aussi été pro-nazie au point d'accepter et de vouloir, pour certains, la création d'une milice (la « milice Perrot ") sous uniforme allemand et dépendante du SD (Sicherheitsdienst). Infime minorité (pas plus de 80 hommes), il n'empêche que l'opprobre en a rejailli abusivement sur l'ensemble du mouvement breton dont l'image en a longtemps été ternie.

2. Les fermes y sont plus modernes (eau courante, motorisation, maison d'habitation, etc.)

3. Le titre complet est en réalité : GRAVIER, Jean-François, Paris et le désert français : décentralisation, équipement, population, Flammarion, 1947, préface de Raoul Dautry, $317 \mathrm{p}$. 
qu'elle est un témoignage de ce " désert ". Cet ouvrage est la première réflexion d'importance en France sur l'aménagement du territoire, réflexion qui s'inscrit dans un contexte plus large de planification incitative à la fois voulue par les dirigeants français et nécessitée par le plan Marshall.

Cette prise de conscience quasi générale concerne tous les domaines; elle conduit à une réaffirmation positive de l'identité bretonne mais avec des ambiguïtés et des limites internes et externes. Dans ce contexte, les dirigeants bretons ont mené auprès des gouvernements successifs une action visant à " rattraper le retard " de la région, mais une région à géographie variable; le découpage administratif créé par le régime de Vichy réduisait la région Bretagne à quatre départements, excluant la LoireInférieure ${ }^{4}$ et par conséquent Nantes, une des deux anciennes capitales du duché de Bretagne. Le maintien de ce découpage conduisit progressivement à n'envisager le travail de lobbying des élus bretons que dans une région réduite à ces quatre départements.

L'action des dirigeants bretons s'est d'abord déroulée dans le cadre d'un organisme indépendant : le CELIB (Comité d'études et de liaison des intérêts bretons), puis dans celui de la région Bretagne, circonscription administrative officielle. Insensiblement s'est construite une région dont l'identité s'affirme reprenant confiance en elle par ses réussites économiques et humaines, mais non sans conflits et reconversions difficiles. Cette évolution s'est réalisée sous l'impulsion des élites locales avec ou contre l'État, puis dans une seconde phase, elle veut s'appuyer sur l'ensemble de la population bretonne et relance les revendications identitaires.

\section{Du constat à l'action ou les années cinquante}

Les années cinquante sont pour la Bretagne des années de reconstruction, mais aussi d'émergence de la modernité, entendue au sens de modernisation économique, mais aussi sociale. La France de la IV ${ }^{e}$ République est aussi celle de l'industrialisation dans tous les domaines (industrie, agriculture, bâtiment), de la planification et des premiers pas de la construction européenne. Cette triple orientation se retrouvait dans la région et trouva un écho dans la volonté d'élites locales elles-mêmes largement renouvelées par le changement politique et par le changement de génération.

\section{Un constat accablant}

Les destructions de la guerre atteignaient les immeubles et les voies de communication; elles étaient certes un handicap mais ont conduit à reconstruire les immeubles avec le " confort moderne" (eau courante, sanitaires, etc.); toutefois, cette reconstruction se fit lentement ${ }^{5}$.

4. Devenue Loire-Atlantique en 1957.

5. Ce n'est qu'à la fin des années soixante que, par exemple, les derniers baraquements disparurent à Lorient. 
Signe de difficultés plus générales, les recensements qui se succédèrent de 1946 à 1962 montrent une Bretagne (4 ou 5 départements) où non seulement l'exode rural était important, mais qui perdait ses forces vives. La natalité et le taux de fécondité restaient élevés. La faim de terres aggrava l'exode rural; celui-ci nourrit certes les villes bretonnes, mais leur industrialisation était insuffisante pour l'absorber; la Bretagne n'attirait pas, faute d'emplois dans les secteurs secondaire et tertiaire. En même temps, Jean-François Gravier souligne que le principal handicap industriel est moins telle ou telle taxe que " la rareté des industriels bretons " et indique qu'il faudrait faire appel à des chefs d'entreprise de l'extérieur ${ }^{6}$. Le mouvement migratoire hors de la région s'accentuait en effet au profit des régions industrielles et de la région parisienne, De manière classique, c'étaient les jeunes adultes de 25 à 35 ans qui émigraient, les jeunes filles étant les premières à partir. Elles ne voulaient plus vivre comme leurs mères dans une maison d'habitation au sol en terre battue, sans eau courante, ni électricité avec tout ce que cela impliquait comme dureté de vie et de conditions de travail; les témoignages abondent, que ce soit dans la presse syndicale agricole ou dans des ouvrages-témoignages postérieurs ${ }^{7}$. La structure de la population active est tout aussi révélatrice : en particulier, le secteur secondaire diminue entre 1954 et 1962 dans la Bretagne à quatre départements (ce n'est pas le cas avec la Loire-Atlantique). Plus largement, la Bretagne était la dernière (ou presque) de la classe pour le revenu par habitant (inférieur de $30 \%$ à la moyenne française), la productivité y était la plus faible de France par habitant, les logements ruraux ou citadins étaient surpeuplés et sous-équipés avec une situation qui allait en se dégradant de l'habitat citadin (lui-même différent d'un quartier à l'autre) à celui du bourg rural et enfin à celui des hameaux des communes rurales.

Encore en 1961, lorsque René Pleven écrit Avenir de la Bretagne ${ }^{8}$, il cite quelques chiffres qui en disent long sur le retard de la Bretagne en 1950 et les débuts d'une évolution.

\section{La volonté politique}

Le constat du retard accumulé dans les équipements publics, dans les domaines industriel, portuaire, touristique, sportif, etc. provoqua une réaction des élus politiques et consulaires qui se devaient d'y mettre fin. Si la situation était devenue dramatique ou perçue comme telle, le contexte de croissance économique générale, le renouveau de la pensée économique

6. GRAVIER, Jean-François, op. cit., p. 253.

7. Cf. les journaux des FDSEA (fédération départementale des syndicats d'exploitants agricoles) comme Le Paysan d'Ille-et-Vilaine et parmi les témoignages, on peut retenir celui de Louis MALASSIS, La longue marche des paysans français, Fayard, 2001, 400 p. ; l'auteur fils de petits paysans du nord de l'Ille-et-Vilaine est devenu ingénieur agronome, professeur et directeur général de l'enseignement supérieur et de la recherche au ministère de l'Agriculture.

8. Calmann-Lévy, 1961, 256 p. 
et sociale favorisant le développement d'un État-Providence, le plan Jean Monnet (1947), l'émergence de la construction européenne, l'avertissement lancé par Jean-François Gravier dans son ouvrage, tout concourut à faire repenser l'avenir de la région en termes neufs dépassant les clivages politiques traditionnels et le catalogue de revendications. Très vite, l'ouvrage de Jean-François Gravier qui contribua à la création d'une Direction à l'aménagement du territoire en 1949, devint la référence des régionalistes et des aménageurs. Il devenait urgent que les responsables régionaux prissent des initiatives.

Ce fut en 1950 que s'ébaucha le CELIB. À l'origine, un homme, Joseph Martray ${ }^{9}$, journaliste, qui publia en 1947 Le problème breton et la réforme de la France. Dès 1948, il lança une revue trimestrielle, Le Peuple breton, qui se présentait comme l'organe du «mouvement breton tout entier " et développait les thèmes de la modernisation, de l'industrialisation, de la construction de l'Europe. Il était en effet président de la fédération bretonne de l'Union fédéraliste des communautés et régions européennes, association qui entendait promouvoir les minorités ethniques autour de l'idée d'un fédéralisme européen ${ }^{10}$. Fédéraliste européen, il était cependant conscient qu'il n'était pas question, vu le passé récent, de demander un statut spécifique pour la Bretagne, mais d'œuvrer pour que celle-ci cessât de décliner et retrouvât la voie du dynamisme.

Cette démarche pro-européenne et fédéraliste signifiait vers 1949/1950 une démarche politique tournée vers l'avenir, prenant en compte le cadre d'un État français centralisé mais qui venait de créer une direction à l'aménagement du territoire. Cette attitude pragmatique se voulait aussi une attitude de dialogue avec l'État sur les points vitaux du développement de la région avec l'appui des élus.

Dans cet état d'esprit, Joseph Martray contacta des hommes politiques de toutes nuances et élus à tous les niveaux (communes, cantons, circonscriptions parlementaires), des représentants des Chambres de commerce, d'agriculture et de métiers, de groupements professionnels. L'année 1949 et le début de 1950 virent l'organisation de plusieurs réunions à caractère inhabituel dans la mesure où elles rassemblaient des élus de partis politiques opposés. Ces réunions précédèrent la réunion constitutive du CELIB qui eut lieu en juillet 1950.

À Quimper, à la veille des fêtes de Cornouaille, le 22 juillet 1950, 150 personnes se rassemblèrent; acceptèrent de siéger côte à côte des représentants du mouvement breton (surtout des associations culturelles), des élus de tous les partis politiques sauf les communistes, des membres des assemblées consulaires et des organisations professionnelles, en attendant les syndicats. Un échec ce jour-là aurait été définitif, mais d'emblée les discours du maire RPF (Rassemblement pour le peuple français) de Quimper,

9. Il fut membre du Comité consultatif de Bretagne pendant le régime de Vichy et il avait été rédacteur en chef de La Dépêche de Brest en 1944 avant la Libération.

10. Cf. Nicolas, Michel, Histoire du mouvement breton, Paris, Syros, 1982, p. 138. 
Joseph Halléguen, et de Joseph Martray précisèrent l'objet de l'organisme à créer. Ils réaffirmèrent clairement le refus du catalogue de jérémiades au profit d'une organisation regroupant le maximum d'élus, de responsables économiques, sociaux, culturels qui pourraient parler et agir au nom de la Bretagne. De cette réunion naquit le CELIB et à la fin de l'année fut élu un bureau provisoire dont la composition reflétait cette volonté œcuménique; présidé par le maire RPF de Quimper, il comprenait deux socialistes, un autre élu RPF, un représentant des organisations professionnelles, un des organisations consulaires et enfin un représentant de l'Association bretonne. Le secrétaire général et véritable animateur en était Joseph Martray; quant au président, Joseph Halléguen, 34 ans, ancien de la France Libre, il était favorable aux idées régionalistes et européennes, fait très rare à l'époque; il marqua profondément les débuts du CELIB, avant sa mort précoce à 38 ans. Afin de doter le CELIB d'un outil de communication, Joseph Martray fit de La Vie bretonne, édition régionale du bulletin fédéraliste, l'organe du CELIB ${ }^{11}$.

Afin de s'affirmer et d'impliquer les élus, le CELIB profita des élections de juin 1951 pour envoyer un programme et un questionnaire aux candidats; s'ils étaient élus, ils devaient s'engager à constituer un intergroupe parlementaire regroupant les élus bretons afin de défendre les dossiers élaborés au CELIB. Tous, sauf les communistes, acceptèrent ${ }^{12}$.

Après une réunion des parlementaires bretons, le 5 août 1951, le CELIB se constitua en association selon la loi 1901; les statuts du CELIB furent déposés le 30 octobre (Journal officiel du 23 novembre 1951). Il devait être financé par les conseils généraux, les communes et les organismes adhérents. Un homme joua un rôle majeur : René Pleven, député des Côtes-duNord, ministre et président du Conseil à plusieurs reprises. La présidence du CELIB lui fut proposée à l'unanimité peu de temps avant qu'il ne redevînt président du Conseil ${ }^{13}$; quatre autres parlementaires devinrent viceprésidents : Joseph Halléguen, député RPF du Finistère, Paul Ihuel, député MRP du Morbihan, André Morice, député radical de Loire-Inférieure et Tanguy Prigent, ancien ministre socialiste de l'agriculture, député du Finistère qui présida la commission parlementaire du CELIB. Cette structure témoignait par sa composition d'une volonté réelle de pluralisme politique et du souhait d'envisager la Bretagne dans ses frontières historiques (les cinq départements) et non dans le cadre régional administratif à quatre départements.

11. CRESSARD, Jean-Pierre, CELIB 1950-2000. Quand la Bretagne s'est réveillée, Spézet, Coop Breizh, 2000, p. 28.

12. Les communistes, selon une lettre de Marcel Hamon, ne pouvaient « travailler en commun avec des hommes politiques qui avaient accepté que le général Eisenhower dispose à sa guise du réduit breton " (allusion à la participation de la France à l'OTAN depuis sa création en 1949), cette prise de position s'inscrivait dans la ligne politique générale suivie par le PCF pendant la guerre froide.

13. Il resta président du CELIB pendant 21 ans. Bougeard, Christian, René Pleven. Un Français libre en politique, Rennes, PUR, 1994, p. 190. 


\section{L'action}

L'existence même du CELIB était une nouveauté et une originalité pour la région et pour la France. Pour la première fois, tous les parlementaires et tous les représentants économiques et sociaux d'une région créaient un organisme transcendant les divisions politiques, leur permettant de se rencontrer afin d'élaborer ensemble les perspectives d'avenir de leur région et de faire des propositions constructives. Non seulement la volonté politique affichée mais aussi les méthodes du CELIB le différencièrent des Comités régionaux d'expansion économique créés ensuite dans toute la France.

L'action du CELIB fut favorisée par la situation politique française dans les années cinquante. En effet, la structure qui fit la force du CELIB sous la IV République était la commission parlementaire du CELIB regroupant les parlementaires bretons appartenant aux cinq départements. Ils se réunissaient tous les mois, parfois plus souvent selon les besoins de l'actualité; ils prenaient position sur des dossiers précis qu'ils allaient ensuite défendre d'une seule voix dans les ministères. Cette structure, particulièrement efficace, dans une situation politique où les gouvernements de coalition ne se maintenaient en place que par quelques voix, formait un véritable groupe de pression breton.

Logiquement, les premiers dossiers concernèrent le monde rural et sa modernisation; cependant pour éviter un effet catalogue, le CELIB s'orienta vers la mise au point d'une planification adaptée à la Bretagne.

C'est dans le cadre du second plan quinquennal (1954-1958) que le CELIB se prépara à agir. Il voulait favoriser la planification régionale avec un programme d'ensemble pluriannuel pour la Bretagne et un véritable engagement de l'État. En juin 1952, le CELIB provoqua une réunion avec les délégués du Commissariat au Plan et de la Direction à l'Aménagement du Territoire, les personnalités des milieux politiques et économiques et les principaux chefs de services administratifs de la Bretagne, mais une première limite à son action apparut : la Loire-Inférieure refusa de participer ${ }^{14}$. Après ces discussions le CELIB put être associé aux décisions nationales. Ce travail, chiffré, aboutit à un véritable " plan breton "; encore fallait-il le faire avaliser par le gouvernement ce qui fut fait moyennant des aménagements. La Bretagne fut ainsi la première à présenter une planification régionale, et pour le second plan quinquennal français, la seule région. Ce fut à la suite de cette initiative et de la popularisation du Plan breton que fut promulguée la loi du 14 août 1954 prévoyant " une organisation régionale, départementale ou locale chargée de mettre au point le développement économique local dans le cadre du Plan "; elle fut suivie en décembre du décret créant les comités régionaux d'expansion économique d'où les parlementaires étaient exclus, à la différence du CELIB, le gouvernement ne voulait évidemment pas risquer une multiplication des lobbies régionaux.

14. Premier signe de la réduction du CELIB aux quatre départements bretons excluant la Loire-Inférieure. 
En Bretagne, l'administration tenta de doubler le CELIB par un comité d'expansion conforme à la nouvelle loi, mais cela échoua et le préfet Jean Benedetti comprit qu'il fallait passer par le CELIB et le fit reconnaître comme comité régional d'expansion économique tout en conservant l'originalité de sa structure avec la présence des parlementaires. Cette reconnaissance officielle fit l'objet d'un décret en octobre 1955.

La Bretagne a bénéficié d'élites locales qui ont su mesurer l'ampleur des difficultés économiques et sociales de la région. Elles ont voulu mettre fin à l'archaïsme de la région, certes : par volonté profonde de modernisation de la part de ces élites? pour conserver l'identité de la région? pour conserver leur emprise sur la population? Ces trois objectifs étaient sans doute au cœur des préoccupations de ces élites dans des proportions variables; leur diversité politique, générationnelle fonde cette complexité. Si leurs objectifs ultimes étaient dissemblables, la nécessité matérielle de construire ou de reconstruire la région dans les années cinquante et au début des années soixante fit taire ces dissonances et permit une union qui resta conjoncturelle. Dès les premiers acquis, cette unanimité de façade vola en éclats.

\section{Nouvel élan et limites}

Le CELIB a eu valeur d'archétype et son exemple a incité à prendre un certain nombre de mesures administratives générales concernant l'ensemble de la France mais bénéficiant aussi à la Bretagne. Une série de décrets en 1954-1955 visèrent à lutter contre la centralisation industrielle, à permettre la création de sociétés d'économie mixte pour aménager et équiper les régions; ces sociétés ont généré à leur tour des sociétés de développement régional pour assurer le financement de ces opérations. Ces mêmes décrets prévoyaient aussi des Programmes d'action régionale (PAR) et la création de région de programme.

\section{Les atouts}

Les atouts de la Bretagne étaient essentiellement humains. La région, on l'a vu, était féconde et la population qui avait émigré, l'avait fait contrainte et forcée, ne trouvant pas d'emplois sur place. Cette population " émigrée " restait en même temps fortement attachée à sa région pour des raisons familiales mais aussi culturelles ${ }^{15}$. Autrement dit, dès qu'elle en aurait la possibilité, cette population s'efforcerait de rentrer au pays.

Un autre atout majeur de la région était une population très scolarisée et plus diplômée que dans la plupart des régions françaises. La rivalité entre les écoles publiques et les écoles privées en est largement responsable, l'impossibilité de se maintenir sur les exploitations agricoles firent que les parents poussaient souvent leurs enfants (les filles en particulier) à obtenir au moins leur certificat d'études voire plus, afin de pouvoir tra-

15. Culture qui ne passe pas forcément par la maîtrise de la langue bretonne. 
vailler en ville, de devenir fonctionnaire ou en tout cas d'avoir une situation stable et correctement rémunérée. Cette stratégie d'insertion sociale et de promotion est confirmée par le fait que les Bretons de la diaspora étaient plus diplômés que ceux restés en Bretagne ${ }^{16}$. La Bretagne fournit donc des employés, des cadres intermédiaires voire supérieurs, tous nécessaires en cas de redynamisation et de modernisation de son économie.

Cette richesse humaine était en quelque sorte consolidée par les réseaux constitués dans la mouvance de l'école laïque ou confessionnelle. Dans ce cadre, la perception d'une région traditionaliste que l'extérieur avait de la Bretagne était due à la forte emprise directe et indirecte que l'Église exerçait. Or, l'Action catholique spécialisée a eu une influence considérable par ses organisations destinées aux jeunes (17-25 ans) telles la JAC (Jeunesse agricole chrétienne), la JOC (Jeunesse ouvrière chrétienne) et la JMC (Jeunesse maritime chrétienne); la première eut le plus d'ascendant en raison de la forte ruralité de la Bretagne. Leur méthode " voir, juger, agir " a amené à une réflexion sur une modernisation équilibrée mais nécessaire pour une meilleure intégration dans l'économie et la société française. La JAC comme la JOC ont aussi mené une considérable action de formation des jeunes filles et des jeunes femmes. Cette action équilibrée envers les deux sexes étaient une nouveauté et elle a permis de donner aux unes et aux autres une formation équivalente, une aspiration à la modernisation en particulier dans les fermes et on sait que celle-ci ait souvent passé par les femmes.

Les atouts humains de la Bretagne sont indéniables et jouèrent un rôle majeur dans son renouveau même si celui-ci rencontra des obstacles.

\section{Limites et obstacles}

Ceux-ci sont d'ordre administratif et économique, mais s'ils ralentirent le processus, ils n'empêchèrent pas des transformations profondes qui font de la région, une région ancrée dans son temps, sans renier, voire en se réappropriant sa culture.

Dès les premiers pas du CELIB, l'ancrage dans l'histoire bretonne se heurta aux conceptions contemporaines en matière administrative et économique. En effet, la question des limites de la région se posa compte tenu de celles de l'ancien duché de Bretagne et des délimitations administratives postérieures à la Révolution française ${ }^{17}$ : avec ou sans la Loire-Inférieure. Or, dans les projets ou réalisations antérieures, la Bretagne historique avait été scindée et réduite administrativement à quatre départements. C'est ce même découpage qui fut repris en 1955 sans concertation. Si ce découpage heur-

16. LAGREe, Michel et PIHAN, Jean, "Les performances scolaires en Bretagne, 1860-1980 ", dans Havinden, Michael A., Queniart, Jean et StANyer, Jeffrey (dir.), Centre et périphérie/Centre and periphery. Bretagne, Cornouailles-Devon, étude comparée/Brittany ans Cornwall \& Devon compared, Exeter, University of Exeter Press, 1991, p. 188.

17. Les premières régions économiques délimitées administrativement l'avaient été dans les années vingt avec le ministre Clémentel, mais sans suite véritable; puis, en 1941, le régime de Vichy créa des régions. 
tait (et heurte encore certains) par rapport à l'histoire de la Bretagne, elle " réglait » d'une certaine manière la rivalité Rennes/Nantes, puisque chacune des deux capitales du duché devenait préfecture régionale, Rennes pour la Bretagne et Nantes pour les pays de Loire. En revanche, le CELIB, malgré son souhait initial et devant les réticences de Nantes et de la Loire-Inférieure avait dû préparer le plan breton dans le cadre d'une Bretagne à quatre départements ce qui la coupait d'une de ses métropoles économiques.

À ces limites administratives, s'ajoutaient des limites économiques et financières. Pour l'État, la région de programme était un cadre mais il n'entendait pas perdre ses prérogatives. Le premier PAR (programme d'action régionale) s'y inséra et la Bretagne, grâce au CELIB, fut la première à pouvoir présenter un plan publié le 13 juillet 1956, mais ce PAR était édulcoré par rapport au programme du CELIB. L'administration centrale restait jalouse de ses prérogatives et il fallut tout l'art de la négociation des préfets de région sur le terrain pour ménager les susceptibilités, mais les limites de la politique gouvernementale apparurent rapidement.

Si le CELIB avait parfaitement chiffré les investissements nécessaires et donné un échéancier, le PAR ne comportait aucune indication de crédit à la fois en raison des difficultés financières du gouvernement français en pleine guerre d'Algérie et des réticences de l'administration centrale. Les orientations principales voulues par le CELIB étaient maintenues (productions agricoles, réorientation des activités maritimes, création de nouvelles industries ${ }^{18}$ ), la définition des "zones critiques " ouvrant droit à des avantages fiscaux et des primes pour les entreprises était très restreinte et excluait la Bretagne centrale c'est-à-dire la zone la plus pauvre au profit des villes. Les limites de ce plan étaient manifestes et classiques : la question budgétaire et les habitudes de centralisation administrative. Mais l'existence même de ce PAR était source d'espoir car il était désormais une référence incontournable quel que soit le gouvernement voire le régime. Les limites de ce plan expliquent la " déception bretonne " et la défense de l'idée d'une loi-programme pour la Bretagne, idée avancée par René Pleven ${ }^{19}$.

Avec la Ve République et l'arrivée comme Premier ministre de Michel Debré, les craintes étaient grandes de voir tomber dans l'oubli ces dispositions, car ses positions très centralisatrices, très jacobines étaient bien connues. Le résultat fut paradoxal et en un sens on peut dire que ce qui dépendait le plus directement de l'État fut accompli et ce qui dépendait le plus de l'initiative privée ne fut que partiellement réalisé. D'une part, les créations d'industrie ne compensèrent qu'à peine, en ce début des années soixante, les licenciements et les fermetures de sites industriels anciens. D’autre part, l'État décida des simplifications administratives et toute la

18. Le plan se gardait bien d'indiquer quelles industries seraient implantées et surtout où elles seraient localisées. En revanche, la construction d'une usine marémotrice sur la Rance était acquise. Elle fut inaugurée en 1966.

19. LAGREE, Michel et PiHAN, Jean, " Les performances scolaires en Bretagne, 1860-1980 ", op. cit., p. 205-256. 
Bretagne devint " zone critique " favorisant ainsi l'implantation d'entreprises nouvelles dans toute la région. Ces décisions furent fondamentales puisque les effets s'en firent sentir après 1962 avec des implantations majeures pour le renouveau de la Bretagne tels par exemple le CNET (Centre national d'études et de télécommunications) à Lannion ${ }^{20}$ et l'usine Citroën à Rennes.

Si la modernisation de la Bretagne voulue par ses élus (politiques, consulaires, syndicaux) rencontra des limites plus que des obstacles infranchissables, se heurtant aux pesanteurs administratives, mais au-delà à celles de l'habitus, elle fut doublement aidée par la rencontre de deux volontés, celle des élus bretons et celle de l'État qui pourtant n'agissaient pas avec les mêmes objectifs. L'État ${ }^{21}$, dans une conjoncture de croissance économique, encourageait partout la modernisation du pays; influencé par la conception keynésienne de l'économie, il mettait à l'ordre du jour la planification (incitative) et l'aménagement du territoire pour mettre fin aux déséquilibres régionaux ${ }^{22}$ et à un risque d'asphyxie de la région parisienne, des choix qui rencontraient une partie des aspirations du CELIB. Celui-ci avait voulu cette modernisation de la Bretagne pour transformer l'image de la région, qu'elle ne soit plus perçue comme archaïque, retardée, etc., mais aussi pour maintenir la population bretonne sur place et faire revenir ceux qui étaient partis. Si le premier objectif correspondait à une volonté d'insertion dans le siècle et dans l'ensemble français voire européen, le second, inégalement exprimé au sein du CELIB était de retrouver l'identité bretonne ou/et de pouvoir l'affirmer sans crainte d'être taxé de "retardé ", bref, sans honte.

La fin des années cinquante et le début des années soixante virent la construction d'équipements de base et la mise en application de mesures qui entraînèrent les débuts de la mutation de la région, de sa modernisation. Celle-ci s'accomplit véritablement, fut visible surtout dans la décennie suivante ${ }^{23}$. Si l'action du CELIB permit ces transformations, en revanche il fallut d'autres facteurs pour l'affirmation d'une certaine identité culturelle.

\section{Un renouveau fondé sur l'identité? ${ }^{24}$}

À partir des années soixante-dix et parallèlement à la re-dynamisation économique de la Bretagne, les Bretons retrouvèrent une certaine fierté; devant la force de la modernisation qui bouleversait profondément le paysage rural et urbain, commençait à se manifester une volonté à la fois de ne pas perdre ce qu'aujourd'hui l'on appelle un patrimoine au sens large

20. Pour plus d'informations sur cette implantation, on peut lire avec profit JeanJacques MonNiER, "Pierre Marzin et la révolution lannionaise (1954-1974) ", Annales de Bretagne et des pays de l'Ouest, avril 1995, p. 87-96.

21. Que ce soit sous la IVe ou la Ve République.

22. Ces préoccupations ne sont pas spécifiques à la France; même si le contexte diffère, elles se retrouvent en Italie vis-à-vis du Mezzogiorno.

23. Dès le recensement de 1968, l'inversion des migrations était sensible.

24. Sur ce thème voir LE COADIC, Ronan, L'Identité bretonne, Rennes, Terre de Brume/PUR, $1998,478 \mathrm{p}$ 
du terme (bâtiments, paysage, mais aussi culture, langue, etc.) et de faire revivre la culture bretonne non pas comme un souvenir folklorique (démarche du XIX ${ }^{\mathrm{e}}$ siècle) mais comme une culture vivante. Pendant ces mêmes années, le mouvement breton resurgit avec un éventail politique assez large, mais ce renouveau suscita ou suscite des malaises, conflits, ambiguïtés qui pèsent encore aujourd'hui.

\section{Recherche d'une identité culturelle}

Les signes extérieurs de l'appartenance à la Bretagne - vêtements, langue - disparaissaient progressivement. Ce fut plus rapide et plus définitif pour les vêtements que pour la langue par exemple. Dès les lendemains de la guerre, dans les zones proches des villes, et au plus tard dans les années soixante, les femmes ${ }^{25}$ avaient totalement abandonné le costume à l'exception de quelques personnes âgées. La langue bretonne elle-même qui n'était parlée que dans la partie occidentale de la région, fut de plus en plus abandonnée après la Seconde Guerre mondiale, non sans des différences notables. En effet, la proportion de personnes qui le parlaient couramment, était très différente selon les classes d'âge et les lieux ${ }^{26}$. L'usage du breton devenait privé et, dans les années soixante, la transmission du breton ne se faisait plus entre les générations; l'interdiction de la pratique du breton à l'école, la volonté de s'insérer dans la modernité (le français y est associé mais pas le breton) ont largement contribué à l'abandon de la langue bretonne ${ }^{27}$ et à une véritable rupture culturelle.

L'abandon n'est certes pas absolu, mais jusque dans les années soixante on peut surtout noter une volonté de maintien, en attendant une période d'adaptation puis de reconquête. En effet, au lendemain de la guerre, les cercles celtiques ${ }^{28}$ se développèrent et, dès 1950 , fut constituée l'association Kendalc'h dont le but était de regrouper en Bretagne tous ceux qui s'intéressaient " au maintien et à la diffusion de la culture et des traditions bretonnes ${ }^{29}$ ". Cette association à buts culturels était en symbiose avec les choix du CELIB jusqu'au milieu des années soixante. Dans les années cinquante-soixante, la culture bretonne était, bon gré, mal gré, une culture maintenue, de conservation d'un patrimoine sans " modernisation ", tout en ayant le souci de donner des références culturelles, identitaires; si cer-

25. Elles ont conservé plus longtemps que les hommes le port du costume breton, en particulier le port de la coiffe, symbole aussi d'appartenance à telle ou telle communauté.

26. C'est ainsi que Léon Fleuriot considère que si 70 à $80 \%$ des personnes âgées connaissent le breton, 5 à 20 \% seulement des jeunes le pratiquent. La pratique du breton se perd; au total vers 1980, 600000 à 700000 personnes le connaissent, mais un tiers de celles-ci peut-être en font un usage quotidien.

27. Sans compter certaines pratiques familiales où quand les parents ne voulaient pas que l'enfant comprenne, on parlait breton devant lui. Cf. LE COADIC, Ronan, op. cit., p. 197-198.

28. Dont la fonction est essentiellement d'apprendre et de faire vivre la musique et la danse bretonne traditionnelles, ils sont aussi des lieux où l'usage du breton est entretenu voire appris.

29. Cf. NiCOLAS, Michel, op. cit., p. 181. 
tains la voulaient nationale, ce n'était pas le cas de la très grande majorité qui entendait seulement ne pas perdre la mémoire d'un passé culturel riche. C'est à la fin des années soixante et dans les années soixante-dix que s'amorcèrent véritablement de nouvelles recherches artistiques, musicales en particulier et une diffusion neuve de celle-ci avec des artistes comme Alan Stivell; il se produisit alors une réappropriation des fest-noz (= fête de la nuit), occasion de se rassembler au son des chants et danses bretons, de s'identifier à une même communauté. Les " années 68 " et le thème " vivre au pays " jouèrent un rôle-clé dans cette période charnière où la Bretagne était devenue une région moderne mais dont les habitants ne voulaient pas perdre leur culture.

\section{Nouveau cours politique et affirmation de l'identité culturelle}

Au cours des années soixante, la Bretagne avait retrouvé progressivement une fierté économique, l'idée d'appartenance au monde moderne, tout en restant attaché à certains modes culturels, particulièrement dans la zone bretonnante (le Finistère, l'ouest des départements des Côtes-duNord et du Morbihan). En même temps, les décisions gouvernementales ne lui donnèrent pas pleine satisfaction et l'environnement idéologique des " années 68 » favorisa le développement de nouveaux courants politiques et de nouvelles revendications d'ordre politique et culturel.

Jusqu'à la fin des années soixante, le CELIB avait réussi non sans peine à maintenir son unité, mais en 1972, René Pleven devint Garde des Sceaux; l'opposition de gauche lui reprocha son ambiguïté et préféra quitter le CELIB. Le bilan de celui-ci n'est certes pas un bilan totalement positif, mais il a permis à la Bretagne de ne pas manquer le tournant de l'industrialisation et plus largement de la modernisation de la seconde moitié du vingtième siècle; en recourant à des experts bretons il a su mobiliser les élites intellectuelles pour préparer les dossiers et démontrer ainsi les ressources matérielles et humaines de la région. Politiquement, il a redonné une cohésion et fait avancer la notion de régionalisation en attendant une décentralisation autre qu'économique. Toutefois, en 1972, les conditions du devenir de la région ne sont plus les mêmes en raison de la crise économique générale, des effets idéologiques et politiques des " années 68 ".

Politiquement le mouvement breton était réapparu en 1957 avec le Mouvement pour l'organisation de la Bretagne (MOB) qui restait encore profondément ancré dans des choix politiques de droite, mais avec l'échec d'une certaine forme de mode d'action politique que représentait le CELIB, 1964 marqua une rupture avec la naissance à l'intérieur du mouvement breton d'un discours de gauche structuré et la valorisation de la situation économique et sociale ${ }^{30}$. Ce discours de gauche, structuré, est symbolisé par la naissance de l'UDB (Union démocratique bretonne) dont certaines préoccupations et revendications rejoignaient la gauche nationale, elle-même

30. Ibid., p. 256. 
en profond renouvellement ${ }^{31}$. Cette évolution montrait que le mouvement breton commençait à se dégager de l'image d'extrême-droite qui lui venait de la guerre. Ces nouveaux militants bretons lui donnaient un visage politique moderne " fréquentable " et ils s'inséraient dans le mouvement des idées fédérales ancrées à gauche.

On voit se dessiner alors deux mouvements, l'un politique, l'autre culturel, qui s'accompagnèrent mutuellement, parfois s'opposèrent, mais ouvrirent une nouvelle voie dans les relations entre l'État et la Bretagne, entre l'appartenance française et l'identité bretonne ${ }^{32}$.

Entre 1972 et la fin des années quatre-vingt-dix, les présidents de la République successifs et leurs gouvernements prirent des initiatives qui eurent souvent un impact sur la reconnaissance de la région (mais toujours dans un cadre non fédéral). La loi du 5 juillet 1972 donnait à la région le statut d'établissement public avec deux assemblées : le conseil régional et le comité économique et social. Le premier est certes composé d'élus, mais au scrutin indirect, et le comité économique et social a des représentants de la vie économique, sociale et culturelle. Le préfet y jouait un rôle majeur.

Toutes les actions menées jusqu'alors par la puissance publique concernaient le domaine économique mais rien n'avait été obtenu sur le plan culturel. Un élan nouveau est donné en février 1977, lorsque Valéry Giscard d'Estaing, président de la République, permit la formulation et la signature d'une charte culturelle adoptée en 1978 par le conseil régional et le comité économique et social. La personnalité culturelle était reconnue par toutes les collectivités territoriales y compris celles de Loire-Atlantique et ceci pour cinq ans; dans ce cadre étaient créés trois organismes : l'Institut culturel, le conseil culturel et une agence technique culturelle devant assurer la logistique des activités culturelles.

Les militants du mouvement breton, en particulier ceux de la mouvance UDB, eurent alors à leur disposition de nouveaux instruments leur permettant de réagir devant la menace de disparition de la langue bretonne ${ }^{33}$ et de la culture qui lui est propre, face à l'uniformisation des modes de vie et de la culture dans l'ensemble de la France et au-delà des frontières. Cette résurgence de la culture bretonne et du régionalisme breton ne s'est pas faite sans réticence des uns, sans esprit de revanche ou d'instrumentalisation de cette renaissance par une fraction nationaliste. Aujourd'hui, à part celle-ci, le travail de mémoire sur le passé, l'acceptation de la réalité historique se font... bien après que les historiens aient mis à bas bon nombre de mythes ${ }^{34}$.

31. Avec le courant de la "nouvelle gauche " et le PSU (Parti socialiste unifié), la création de la FGDS (Fédération de la gauche démocratique et socialiste) en attendant la refondation du PS en 1971.

32. Relations fort diverses et mélange franco-breton au « dosage " très variable.

33. Ils s'efforcèrent de développer l'enseignement de la langue bretonne, les publications en breton, les spectacles en breton, de créer des organismes culturels spécifiques.

34. Comme le démontre le colloque qui s'est tenu à Brest en novembre 2001. Les actes 
L'affirmation de l'identité bretonne n'a pu se faire avec une représentation positive que lorsque la région est sortie du marasme économique, ce que le CELIB a permis partiellement de faire mais avec l'aide de l'État et le soutien des élites régionales installées dans la région ou en " exil " dans la région parisienne. Les années soixante-dix ont permis un renouveau culturel tandis que des décisions politiques nationales accompagnaient celui-ci.

Un pas décisif a été franchi, lorsque, après l'élection de François Mitterrand à la présidence de la République et l'arrivée de la gauche au pouvoir en 1981, la loi Defferre fut promulguée; elle mettait fin à la tutelle des préfets sur le conseil régional et les conseils généraux, l'exécutif étant entre les mains des présidents de région et de conseil général; les conseillers régionaux sont désormais élus au suffrage universel direct. La région pèse certes plus lourd que par le passé mais la France reste un pays qui ignore le fédéralisme et préfère la notion de décentralisation.

Aujourd'hui, la Bretagne est confrontée aux critiques vis-à-vis d'une agriculture moderne polluante (les nitrates par exemple); elle doit aussi veiller à ne pas gâcher les acquis culturels en évitant les dérives nationalistes et bretonnisantes "impérialistes".

\section{RESUME}

Au lendemain de la Seconde Guerre mondiale, une partie de la population bretonne prend conscience de son retard économique. Les dirigeants bretons ont créé un organisme de réflexion et d'action : le CELIB (Comité d'études et de liaison des intérêts bretons). Celui-ci a été un instrument de lobbying auprès de l'État. Cette action, conjuguée à celle de l'État, favorisera la modernisation économique.

Celle-ci a permis à la région d'avoir de nouveau une image positive d'ellemême. Elle a pu alors renouer avec l'affirmation de son identité culturelle.

\section{ABSTRACT}

Just after the Secund World War, part of the Breton population became aware of the fact that they were economically behind. So the Breton leaders decided to create a reflexion and action institution: the CELIB (Comité d'études et de liaison des intérêts bretons). It soon appeared as an instrument of lobbying to the government. Its action, alongside with the State's, favoured the economic modernization.

With this development, the region had a better self-image. Consequently, she had the opportunity to assert its cultural identity.

du colloque ont été publiés sous la direction de Christian BOUGEARD, Bretagne et identités régionales pendant la Seconde Guerre mondiale, Brest, Université de Bretagne occidentale, CRBC, 2002, $409 \mathrm{p}$. 